\title{
Una aproximación al análisis de Redes egocéntricas de colaboración interinstitucional
}

\author{
M Antonia Ovalle-Perandones, Carlos Olmeda-Gómez, Antonio Perianes- \\ Rodríguez - Universidad Carlos III de Madrid ${ }^{1}$
}

\begin{abstract}
Resumen
¿Es posible establecer la red de influencias entre instituciones involucradas en la producción científica? ¿Comparten los nodos de las redes de influencias interinstitucionales características sectoriales? ¿Resulta interesante profundizar en las redes egocéntricas de colaboración con datos de coautoría? Estas tres preguntas de investigación han sido las que han guiado el desarrollo de este artículo y para responderlas en la primera parte del trabajo se analiza el fenómeno de la colaboración medido a través de la coautoría, revisando además cuál o cuáles son las aportaciones que el análisis de redes egocéntricas realiza frente a un análisis de redes sociales completo. A continuación se propone el uso de las redes egocéntricas para una mejor comprensión de la colaboración científica y de la red de influencia entre instituciones con producción en coautoría científica en el área de Química en España.
\end{abstract}

Palabras clave: Colaboración científica - Coautoría - Redes egocéntricas

\begin{abstract}
Is it possible to establish the influence network of institutions involved in scientific production? Do you share the nodes of the influence network sectorial characterics? What is the advantage to deepen on egocentric collaborative networks with coauthorship data? These three research questions have been those that have guided the development of this article and to answer the first part of the paper analyzes the phenomenon of collaboration as measured by coauthorship are discussed in which one or what are the contributions of network analysis egocentric done before a complete social network analysis. We propose the use of egocentric networks for a better understanding of scientific collaboration and the influence network among institutions with scientific coauthorship in Chemistry area in Spain.
\end{abstract}

Key words: Scientific collaboration - Coauthorship - Egocentric networks

\footnotetext{
${ }^{1}$ Enviar correspondencia a: M Antonia Ovalle, movalle@bib.uc3m.es
} 


\section{El fenómeno de la colaboración científica medido a través de la coautoría}

En la ciencia moderna la colaboración, formal o informal, es una de sus características esenciales. Ante este hecho confirmado, del término colaboración se han aportado múltiples definiciones no consensuadas cuyo eje común en todas ellas gira en torno al trabajo conjunto desarrollado para que los agentes implicados logren unos objetivos comunes y alcancen un conocimiento compartido (Katz JS y Martin BR, 1997), (Hara N et al., 2003). Además de esos motivos, la colaboración implica la idea del beneficio mutuo y de una relación bien definida, pero siempre sin abandonar la idea de una consecución común de propósitos que se comparten por todos los agentes implicados en los procesos colaborativos (Mattessich PW y Monsey BR, 1992).

Ante la ausencia de una definición única de colaboración, la aproximación al concepto se complica también por el uso de términos que en ocasiones se emplean de forma similar para identificar conceptos diferentes. Por ejemplo, es lo que sucede con el término colaboración que se utiliza indistintamente tanto por contacto, cooperación o coordinación (Mattessich PW y Monsey BR, 1992). Por contacto se entiende el intercambio de información para alcanzar un beneficio mutuo, caracterizada esta relación por un nivel inicial de confianza y compromiso, más habitual entre individuos que entre organizaciones. El concepto de cooperación o interconexión, se caracteriza por la existencia de contactos y relaciones informales que existen sin que se defina una misión, una estructura o se planifiquen los esfuerzos. La información se comparte a medida que se necesita, mientras que la autoridad permanece en cada una de las organizaciones. En este caso los resultados y los beneficios quedan al cuidado de quien los obtenga puesto que no son compartidos. El concepto coordinación refleja un proceso que supone la comunicación, la planificación y el reparto de recursos, riesgos y recompensas, con el propósito de lograr eficiencia y efectividad para alcanzar unas metas comunes fijadas de antemano por las organizaciones involucradas. La autoridad permanece en cada una de las organizaciones, aunque la asunción de riesgos supone también el reparto de responsabilidades. El concepto de colaboración denota en sí un tipo de relación más duradera e influyente. Provoca que las organizaciones que desarrollan sus actividades de forma separada, se organicen en una forma de trabajo radicalmente nueva con el fin de alcanzar los cometidos comunes. Estas relaciones exigen de una planificación integral y en este caso la autoridad se determina por la 
propia estructura que tenga el proceso de colaboración (Perianes Rodríguez A et al., 2010).

De entre todas las posibles formas de abordar la colaboración científica, la coautoría en publicaciones es ampliamente utilizada como una medida apropiada para el estudio de la colaboración (Bordons M y Gómez I, 2000). Por coautoría se entiende la aparición conjunta de dos autores como responsables de un documento (White HC y McCain KW, 1997), si bien no todas las colaboraciones científicas conllevan necesariamente la publicación de artículos coautorados, ya que pueden obtenerse materializarse con forma diferente y dar a conocer los resultados (outputs) en colaboración a través de la coautoría en monografías, en trabajos presentados a congresos, en solicitudes de proyectos 0 en informes de investigación, junto a la coautoría en el ámbito tecnológico de patentes o modelos de utilidad, si bien el concepto de autor en estos documentos tiene sus connotaciones, ya que no se recogen como tal, sino que las intervenciones son como solicitantes o inventores. Además es necesario añadir que en ocasiones las colaboraciones pueden no siempre se ven finalmente reflejadas en documentos coautorados (Laudel G, 2002), (Luukkonen T et al., 1992), (Melin G y Persson O, 1996), (Subramanyam K, 1983).

La coautoría de un artículo, como forma de asociación en la que dos o más científicos de manera conjunta informa de los resultados de la investigación que han desarrollado sobre algún tema, es el indicador más visible de la colaboración, por lo que se utiliza con frecuencia para medir de forma aproximada la actividad de colaboración (Russell J M et al., 2009), además es la unidad básica de análisis en los estudios bibliométricos y más recientemente en los análisis de redes de colaboración científica (Milojevic S, 2010). Aunque la coautoría solo mide una parte de un fenómeno amplio y complejo, esta medida en ocasiones es la única de la que se disponen datos controlados, cuantificables y extraíbles a diferentes escalas por procedimientos automáticos que se aproximen al estudio de la colaboración científica. Otro tipo de evidencias de colaboración, por ejemplo son las mantenidas en conversaciones, prestación de apoyo, compartición de técnicas o datos, etc., pero que no dejan señal en las publicaciones, salvo las recogidas en los agradecimientos de los trabajos (Cronin B, 2001). Por lo tanto es obligatorio aclarar que con la coautoría quedan cuantificadas tan solo una parte de las colaboraciones posibles (Katz JS y Martin BR, 1997), (Laudel G, 2002), (Smith D y Katz JS, 2000) siendo la unidad de medida ampliamente aceptada y utilizada por la comunidad que desarrolla estudios informétricos. 
Teniendo en cuenta las consideraciones que se han aclarado y que afectan a la unidad de análisis empleada, la coautoría en este trabajo se utilizará como sinónimo de redes de colaboración y se realiza el estudio de las redes egocéntricas de algunas de las instituciones involucradas en la producción científica en colaboración dada un área determinada. Inicialmente esas redes egocéntricas se establecen a partir de la selección del vecindario inmediato de las instituciones españolas con producción en el área ANEP $^{2}$ Química. Con ello se pretende disponer de la red de influencia de dichas instituciones, estudiando la estructura que resulta y los puntos vinculados a la realidad registrada en las redes de publicaciones científicas (Molina JL et al., 2001).

\section{La aportación de las redes egocéntricas}

La revisión histórica de la literatura del análisis de redes sociales muestra dos enfoques diferentes, de un lado el sociocéntrico y de otro el egocéntrico (Wellman B, 1988), (Molina J L, 2005). La aproximación sociocéntrica explica las propiedades de un grupo de conexiones existentes entre un grupo de nodos definidos previamente por la existencia de una entidad que ocurre previamente o por un criterio que se indica por el investigador. En el estudio sociocéntrico o de redes completas, se incluye en el conjunto actores las relaciones conocidas que ocurren entre ellos, así en el fenómeno que representan las redes es necesario conocer o suponer aspectos relevantes de las relaciones entre sus actores. Mientras que en la aproximación egocéntrica, también denominada de influencia o redes personales, parte de las conexiones se trazan a partir del denominado ego o actor específico y en aquellos actores con quien se relaciona éste (Carrasco J A y Miller EJ , 2006).

Después de conocer los dos tipos de enfoques en el análisis de redes sociales, se define una red, bien sea completa o egocéntrica, como un grafo y la información adicional de los vértices, nodos o actores, junto a las líneas que los vinculan en ese grafo (Nooy W et al., 2004). Las razones que justifican el estudio de redes egocéntricas se basan, no en mostrar todo el reflejo del universo de la red, sino en extrapolar la perspectiva reflejada en la visón heliocéntrica de los individuos centrales en ese universo y sus relaciones, como un todo (Moya Anegón $F$ et al., 2005).

\footnotetext{
${ }^{2}$ La Agencia Nacional de Evaluación y Prospectiva (ANEP) pertenece al Ministerio de Ciencia e Innovación. Este organismo organiza las diferentes líneas de investigación de la comunidad científica y tecnológica en veintiséis áreas temáticas, teniendo presentes las tendencias internacionales
} 
En las redes egocéntricas, a los nodos centrales de ese universo que también se les denomina egos, pueden ser personas, grupos u organizaciones y el enfoque facilita una imagen buena, además de fiable, de las redes, al menos en cuanto a los vecindarios locales, en los que están incluidos los egos. De ellos se pueden conocer el número de nodos conectados, la intensidad de los vínculos que ocurren entre ellos y alguna característica cualitativa de los nodos. Este enfoque egocéntrico a su vez muestra el conjunto de datos con una perspectiva de análisis micro, siendo esas áreas locales una muestra de la gran red en la que participan las instituciones. El método egocéntrico como ya se ha dicho se orienta a recopilar información sobre los vínculos entre los actores a los que se conecta. Esta información es útil para entender cómo las redes afectan a los nodos y a su vez es una imagen, por supuesto incompleta, del entramado de la red como un todo (Hanneman RA, 2005).

Las redes egocéntricas van a estar basadas en la relación de los egos con sus vecinos a una distancia geodésica de uno, pudiéndose ampliar el análisis del vecindario a distancia geodésica de dos. En el caso del vecindario del ego a distancia uno, lo que ocurre es que se están representando las relaciones con su vecindario más inmediato. Cuando se muestren las redes de relaciones a distancia dos, son las relaciones con sus vecinos inmediatos (distancia uno) y junto a ellas las que ocurren con el vecindario de sus vecinos, de tal modo que incluyen además las relaciones mediadas por los nodos inmediatos.

Las redes egocéntricas de las instituciones, se entienden como la red sobre la que una institución ejerce o puede ejercer cierta influencia y puede ser objeto a partir de la teoría estructural de la influencia social (Friedkin NE, 1998). Howard D. White aplicó estos principios a redes de citación, con la idea de generar de cada autor con publicaciones científicas, lo que denominó análisis CAMEOs, que consistirían en la generación de redes de "identidades de citación" compuesta por todos aquellos autores citados por un autor focal, la generación de redes de "creadores de imagen de cita" integrada por aquellos que han citado a un determinado autor central y la “imagen de citación" derivada de la cocitación de autores con un determinado autor focal (White HC, 2001). Propuso además, que las redes de coautoría también podían formar parte de los análisis CAMEOs personales. En este caso, se sigue la misma lógica, pero realizando una propuesta de análisis, a partir de redes de colaboración institucional y no personal.

La visualización de redes completas se enfrenta en ocasiones a los límites tecnológicos y aunque técnicamente sea posible, su uso no permite una interpretación fácil del resultado que la red toma con forma de grafo, por lo que se 
incumple el objetivo final de cualquier representación. Es por ese motivo que en la teoría de redes sociales se han propuesto diferentes métodos para comprender los aspectos fundamentales de una red, en ocasiones con matices parciales, pero centrándose en los aspectos más importantes de la misma. Con el fin de lograr el objetivo de las visualizaciones, se pretende que éstas hagan visibles y comprensibles aquellos fenómenos de la realidad que de otro modo no podrían ser accesibles por el ojo humano, especialmente en aquellos que no tienen una naturaleza visual. Así la acción de visualizar consiste en transformar datos de naturaleza abstracta y hechos complejos de la realidad en mensajes visibles que hace que la información que subyace oculta en ellos sea comprensible para los humanos (Costa J, 1998).

\section{Aspectos metodológicos}

De entre las metodologías propuestas para la visualización de un dominio científico, en este trabajo el dominio de la Química, se va a aplicar la propuesta por Katy Börner y sus colegas (Börner $K$ et al., 2003). Es ésta una metodología constatada como adecuada para la visualización y el análisis de dominios científicos (Vargas Quesada B et al., 2008). La propuesta comienza con lo que afecta a la extracción de datos de coautoría entre instituciones, que se ha realizado utilizando algunos productos de la empresa Thomson Reuters, en particular utilizando el portal Web of Science (WoS).

El WoS es una fuente de información ampliamente utilizada en estudios de naturaleza informétrica y apropiada para profundizar en la coautoría científica, sin olvidar todos los aspectos ya mencionados en cuanto a esta unidad de medida. Los motivos principales del uso de esta fuente son dos. Por un lado la cobertura de las revistas incluidas en los índices que es además de internacional, un recurso multidisciplinar, en cuanto a su amplia y diversa cobertura científica. Junto a ella, cabe destacar el potencial de la información que queda indizada en los registros, tanto por el control realizado para las instituciones a las que se vinculan todos los autores que firman los trabajos indizados, como con el almacenamiento de las referencias bibliográficas de los trabajos que se indizan, proceso que permite el desarrollo de análisis de citación entre trabajos. Otras fuentes de información con una naturaleza similar no siempre indizan todos los autores de un documento, siendo éstas desechadas para cualquier estudio de autores científicos. Estos motivos, llevan a utilizar el WoS como fuente de datos pertinente para el análisis de cualquier dominio científico, pero en particular para el caso de la Química se ha 
demostrado que los índices de citas, tan solo una parte de los recursos disponibles en el WoS, se alcanza un porcentaje sobre el total de la cobertura temática de Thomson Reutres que la sitúa entre las tres primeras disciplinas a las que se les da una mayor cobertura (Moed HF, 2005).

Los índices de citas utilizados son el Science Citation Index Expanded ( $\mathrm{SCl}$ Expanded). Junto a esta base de datos integrada en el Web of Science también se ha extraído información de los Journal Citation Reports, en su edición Science Edition. Para desarrollar este trabajo se han trabajado con todos los registros de la producción científica española recogida en el WoS en septiembre del año 2007, siempre que en los registros se haya recogido el término "Spain" en el campo dirección institucional, que se hayan publicado entre los años 1995 y 2005, siendo 304161 los documentos que cumplen esos criterios.

La relación entre las categorías establecidas por Thomson Reuters y las áreas ANEP para el caso de la Química, se establece con las categorías que se enumeran a continuación: Chemistry, Applied; Chemistry, Multidisplinary; Chemistry, Analytical; Chemistry, Inorganic \& Nuclear; Chemistry, Organic; Chemistry, Physical; Education, Scientific disciplines; Electrochemistry; Engineering, Environmental; Environmental Sciences; Toxicology.

La unidad de análisis basada en instituciones se medirá con datos de coautoría. La elección de los nodos focales se ha realizado a partir de la obtención de una batería de indicadores en el que se incluyen indicadores bibliométricos (citas y producción científica), junto con otros derivados del análisis de redes y la obtención de diferentes medidas de centralidad: grado, grado entrante, grado saliente, cercanía, intermediación (Wasserman S y Faust K, 1994), carga (Brandes U, 2008), pagerank (Brin S y Page L, 1998), comunidad (Fenn DJ et al., 2009), eigenvector (Bonacich P, 1972), hub y autoridad (Kleinberg J M, 1998).

Si bien inicialmente las relaciones de colaboración parten de la premisa de ser relaciones recíprocas, en las que la institución a colabora con la institución b y sin excepción eso supone que se puede afirmar que la institución b colabora con la institución a, para el cálculo de muchos indicadores de redes y por la importancia de su cálculo, se calcula con las relaciones de coautoría la Tasa de Colaboración Asimétrica (TCA) para medir la importancia de unas relaciones con respecto a otras. La TCA es una adaptación del Índice de Afinidad enunciado por Zitt (Zitt M et al., 2000), que se concibe inicialmente para medir relaciones asimétricas entre dos países y se ajusta sobre todo, para resaltar la importancia científica de los países socios sobre el total de la coautoría. 
Adaptando el concepto del Índice de Afinidad, a las instituciones como unidad de análisis, hay que señalar que la TCA es una media del total de la colaboración entre una institución a y otra b, comparado con el total de la colaboración de la institución a con el resto de instituciones. Naturalmente esa tasa de colaboración de la institución a con la b, o de la institución b con la a, está influida por el tamaño global de la colaboración con el resto de instituciones. De ese modo la relación entre ambas instituciones pasa de ser una relación simétrica a una relación asimétrica y las aristas iniciales se convierten en arcos, para representar la importancia de los vínculos que unen a los diferentes nodos (en los casos que nos ocupan, instituciones) teniendo en cuenta la relación con el total de nodos con los que se vinculan dichas instituciones (Olmeda Gómez C et al., 2008).

$$
\begin{aligned}
& \operatorname{TCA}\left(\text { Inst }_{a} \rightarrow \text { Inst }_{b}\right)=\frac{C O L\left(\text { Inst }_{a} \leftrightarrow \text { Inst }_{b}\right)}{\operatorname{COL}\left(\text { Inst }_{a} \leftrightarrow \text { total }_{\text {ins }}\right)} \times 100 \\
& \operatorname{TCA}\left(\text { Inst }_{b} \rightarrow \text { Inst }_{a}\right)=\frac{C O L\left(\text { Inst }_{b} \leftrightarrow \text { Inst }_{a}\right)}{\operatorname{COL}\left(\text { Inst }_{b} \leftrightarrow \text { total }_{\text {ins }}\right)} \times 100
\end{aligned}
$$

Una vez que se tiene la información de los trece indicadores mencionados para todos los nodos de la red, la dimensionalidad de la matriz resultante derivada del uso del grupo de variables se reduce ejecutando un Análisis Factorial de Componentes Principales con el fin de descubrir si comparten alguna estructura latente, no observable directamente (Cea D'Ancona MA, 2002). Para finalizar los aspectos metodológicos, por lo que se refiere a la representación gráfica, se selecciona la técnica de visualización que se ajusta al modelo de distribución espacial conocido como spring-embedder (Eades $P$, 1984).

\section{El dominio científico de la Química en España}

Existen estudios previos que han analizado la producción científica española por las diferentes especialidades, con coberturas cronológicas diferentes empleando datos que han sido extraídos de las fuentes de obtención de datos empleadas en este estudio, confirman la buena posición de la Química en el contexto nacional. En el año 2003 la Comisión europea publicó un informe utilizando indicadores de Ciencia y Tecnología, con una cobertura temporal que comprende los años incluidos entre 
1995 y 1999, en el que también la producción en Química representa el 16,3\% sobre el total de la producción científica española (European Comission, 2003).

En los informes elaborados por grupos de investigación españoles también se confirma la posición del área. Así en el año 2004 la investigación desarrollada en el seno del CSIC, por investigadores actualmente vinculados al Instituto de Estudios Documentales sobre Ciencia y Tecnología (IEDCYT) (Gómez I et al., 2004), con un análisis la producción científica española durante los seis años comprendidos entre 1996 y 2001, si bien se incluyen otras bases de datos (ICYT para las revistas nacionales y se estudia la producción científica tecnológica), deja constancia de la importancia de la producción científica en revistas internacionales en Química y se posiciona en tercer lugar con un $\mathbf{1 8 . 6 \%}$ acumulado sobre la producción científica de España en todo el periodo.

En diversos trabajos desarrollados por componentes del grupo de investigación Scimago con una cobertura temporal que comprende desde el año 1990 al 2004 (Moya Anegón F (dir.) et al., 2007), para la producción comprendida entre 1995 y 2003 (Moya Anegón F y Chinchilla Rodríguez Z, 2005) y aquella ocurrida entre los años 1998 y 2002 (Moya Anegón F y Chinchilla Rodríguez Z, 2004), de nuevo confirman que la Química se encuentra entre las áreas temáticas que acumulan mayores valores de producción a nivel nacional. Y en la tesis doctoral desarrollada por uno de los componentes de ese grupo y que analiza el dominio científico español entre los años 1995 y 2002 (Chinchilla Rodríguez Z, 2004), la Química aparece como uno de los campos temáticos que está por encima de la media mundial en impacto y durante esos años es la tercera área con mayor producción en términos absolutos, representando un $12.33 \%$ sobre el total de la producción española.

La producción científica española entre los años comprendidos desde el 1995 al 2005 en el área ANEP Química, supera los cuarenta y nueve mil documentos (un total de 49237) que sobre el total de producción nacional representan el 16,19\%. Tan solo tienen más peso que la Química, las áreas ANEP Medicina, con un 33,64\% y la Biología Molecular, Celular y Genética, con un 16,46\%.

La producción en el área Química se caracteriza por el dominio de los artículos sobre el resto de tipos documentales, así 46405 artículos se recogieron en las bases de datos ISI dentro de este área temática cuando España aparece al menos en una ocasión mencionada en el campo Address de las base de datos WoS. Es un área temática con un potencial investigador de 55291,56 y un FITN de 1,19, siete décimas superior al impacto medio de la producción total española en el mismo 
periodo. Se puede afirmar que el área Química tiene un buen impacto, siempre y cuando que ese impacto sea medido con las citas observadas. La producción total de documentos recibe un total de 83355 citas, lo que supone que cada documento producido en este área (49237) recibe una media de 1,69 citas, con un índice de coautoría medio en el periodo es de 4,48 autores.

En un nivel de autoría diferente, la institucional, en Química ocurre que aproximadamente la mitad del total de los documentos publicados no se realizan en colaboración entre instituciones (colaboración interinstitucional). Del conjunto de documentos en los que sí ocurre la colaboración entre instituciones, una parte importante se concentra en documentos en colaboración interinstitucional internacional, es decir, aquellos documentos producidos en el área y que son firmados por instituciones ubicadas en más de un país, además de una institución española. Sería el caso de más de quince mil documentos producidos en Química durante el periodo cronológico analizado. Entre los otros cuatro tipos de colaboración (intrasectorial, intersectorial, regional y nacional) se produce una aproximación en los valores dos a dos. El número de documentos en colaboración entre instituciones de diferentes sectores (intersectorial) y documentos en Química en colaboración dentro de una única comunidad autónoma (regional), toman valores próximos a los ocho mil ochocientos documentos. De igual modo la colaboración entre instituciones de un solo sector (intrasectorial) y documentos entre instituciones de dos o más comunidades autónomas diferentes (nacional), para la producción química española, se superan los cuatro mil setecientos documentos.

\section{Las redes egocéntricas de coautoría interinstitucional}

Con los datos de coautoría entre instituciones españolas, se elabora la red de colaboración entre las empresas con producción española con visibilidad internacional en Química y el resto de instituciones españolas. Esta red es de modo 1 ya que está compuesta por un único conjunto de nodos, formado por instituciones vinculadas a diferentes sectores. Las relaciones se reflejan en una matriz asimétrica y ponderada, fruto de aplicar la ya argumentada TCA a cada par de relación se le otro un peso en la relación que será diferente para los nodos. Esta red además tiene un tamaño medio (Börner $K$ et al., 2007) y está formada por un total de 463 instituciones, poco densa, ya que la relación entre el número de relaciones dirigidas existentes y el número de relaciones dirigidas posibles alcanza un 0.0078 , una 
densidad baja ya que de entre las posibles relaciones de coautoría que podrían ocurrir en la red, no se materializan ni tan siquiera el $1 \%$ de ellas.

La adscripción sectorial de las 463 instituciones que integran la red aparece desglosada en la tabla 1. Como en el párrafo previo se indica que la red está centrada en las relaciones de coautoría de las empresas con otras instituciones, ese sector concentra el mayor número de nodos, un total de 294. En esa misma tabla también se incluye la forma con la que cada institución quedará representada, en función de la adscripción de los nodos a un determinado sector.

\begin{tabular}{|l|c|c|}
\hline \multicolumn{1}{|c|}{ Sector } & Forma & Nodos \\
\hline Administración & & 37 \\
\hline EPI & & 5 \\
\hline Sistema Sanitario & & 30 \\
\hline Sistema Universitario & - & 47 \\
\hline Empresa & - & 294 \\
\hline CSIC y centros mixtos & $\mathbf{D}$ & 45 \\
\hline Otros & $\boldsymbol{V}$ & 5 \\
\hline
\end{tabular}

Tabla 1. Adscripción sectorial de las instituciones

Después de realizar el Análisis Factorial mencionado en el apartado que explica los aspectos metodológicos de este artículo, se centrará el análisis de las redes egocéntricas en tres instituciones, aquellas que alcanzan mayores valores en el primer factor, una vez que se ha realizado la rotación Varimax. Las tres instituciones, vinculadas al mismo sector, son la Universidad de Barcelona (UB), la Universidad Autónoma de Barcelona (UAB) y la Universidad Complutense de Madrid (UCM). A continuación se analizan las redes egocéntricas de esas instituciones, en las que la forma de cada nodo que pertenece a los vecindarios inmediatos refleja el sector institucional al que pertenece y el color de los enlaces revelará la intensidad de las relaciones, según lo calculado por la TCA, quedando identificados con tonos azules los valores entre 1 y 49, mientras que los enlaces con más peso tienen tonos verdes, con unos valores entre 50 y 100 . Una baja TCA entre dos nodos, indica que mantienen relaciones con otros nodos. Frente a valores altos de la TCA entre dos nodos, reflejan que ambos mantienen escasas relaciones ajenas a la relación entre ellos. En el Anexo (tabla 2) se incluye la relación de abreviaturas de todas las instituciones incluidas en las representaciones. 
En los párrafos posteriores se va hablar de qué nodos son dependientes en las tres redes. Un nodo dependiente es aquel nodo que solo comparte enlaces o que solo se relaciona con un único nodo, en éste caso por lo tanto que solo se relaciona con el ego.

La red egocéntrica de la Universidad de Barcelona (UB) con su vecindario inmediato, nodos que se sitúan a una distancia 1 del ego, aparece en la figura 1 . La red está formada por 62 nodos, lo que supone en términos porcentuales el 13.39\% de la red completa de colaboración entre empresas españolas e instituciones españolas durante el periodo 1995-2005, unidos esos nodos por un total de 240 relaciones. Las relaciones que establece el ego UB de forma general tienen más peso cuando se establecen con empresas, nodos con forma de rombo, que con nodos de otros sectores institucionales.

Además, en la red egocéntrica de la UB, siendo la institución focal una institución del sector universitario, representada con forma de círculo, se puede observar la alta frecuencia de los nodos del sector empresas con respecto a la aparición de nodos con otras formas, es decir, de otros sectores. De todos ellos, son dependientes del nodo ego UB un total de catorce instituciones y son: Asistencia Tecnológica Medioambiental SA (ATMSA), Química Farmacéutica Bayer SA (BAYERB), Ekolur Asesoría Ambiental SL (EKO), Euromed SA (EUROM), Centro de formación IUSC (IUSC), Laboratorio Lasa SA (LASA), Laboratorio Lesvi SL (LESVI), Lucta SA (LUCTA), MacDermid Española SA (MCDERM), Monocrom SL (MONOC), Laboratorios Ordesa (ORDESA), Prema Compañía SA (PREMA), Pymag SA (PYMAG), Valenciana de Cementos (VALCEM). Teniendo en cuenta la formade estos catorce nodos en la imagen de la red y la información que se puede extrapolar por la información los nombres de las instituciones, los nodos dependientes de la UB son nodos que pertenecen principalmente al sector empresas. 


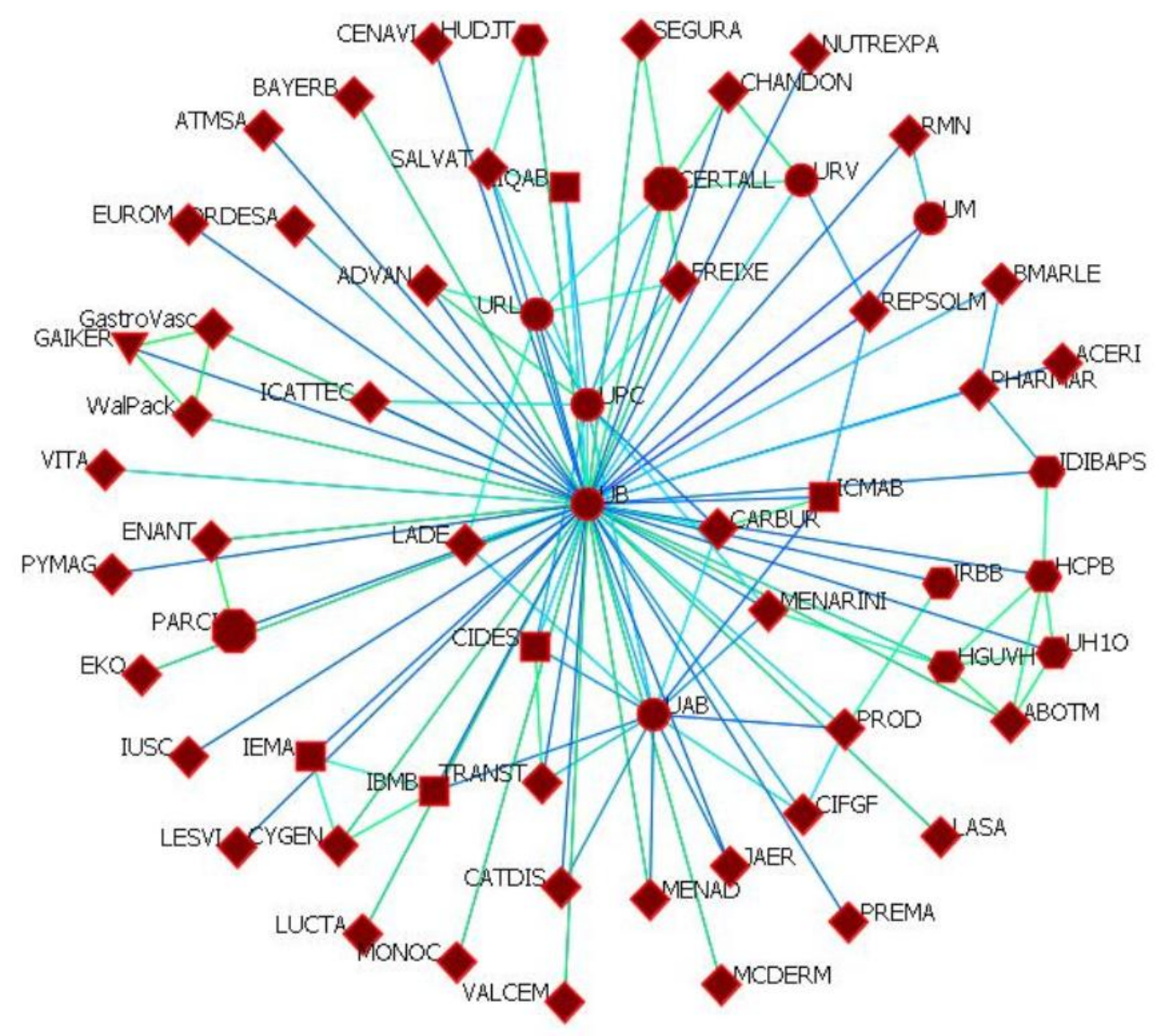

Figura 1. Red egocéntrica de la UB

La red egocéntrica de la Universitat Autónoma de Barcelona (UAB) se representa en la figura 2 con las relaciones que se establecen con sus nodos inmediatos. Para el caso de la UAB ese grupo está formado por 43 nodos, lo que supone en términos porcentuales el $9.29 \%$ sobre la red completa de colaboración entre empresas españolas e instituciones españolas durante el periodo que abarca este artículo, unidos por un total de 170 relaciones. En la red de este ego, también ocurre que de forma general las relaciones tienen más peso cuando la UAB se relaciona con empresas frente al vínculo con instituciones de otros sectores.

Si bien la $U A B$ es una institución universitaria, en su red egocéntrica hay una alta frecuencia de nodos del sector empresas. Entre todo el vecindario inmediato de la $U A B$, son nodos dependientes del ego ocho instituciones que no tienen relación en la red con otros nodos diferentes a la UAB. Sus nodos dependientes son: Asfaltos Españoles SA (AESA), Biosensores SL (BIOSENS), Boehringer Ingelheim España SA (BOEHRB), ICI Spain SA (ICI), Labiana Life Scientific SA (LABIANA), Lipotec SA (LIPOTEC), Centro de investigación J Uriach \& Cia SA (URIACH), Witco España SL (WITCO). Como se puede apreciar en la lectura de este grupo de denominaciones 
de las instituciones, todas son empresas, con forma de Sociedad Anónima (SA) o Sociedad Limitada (SL).

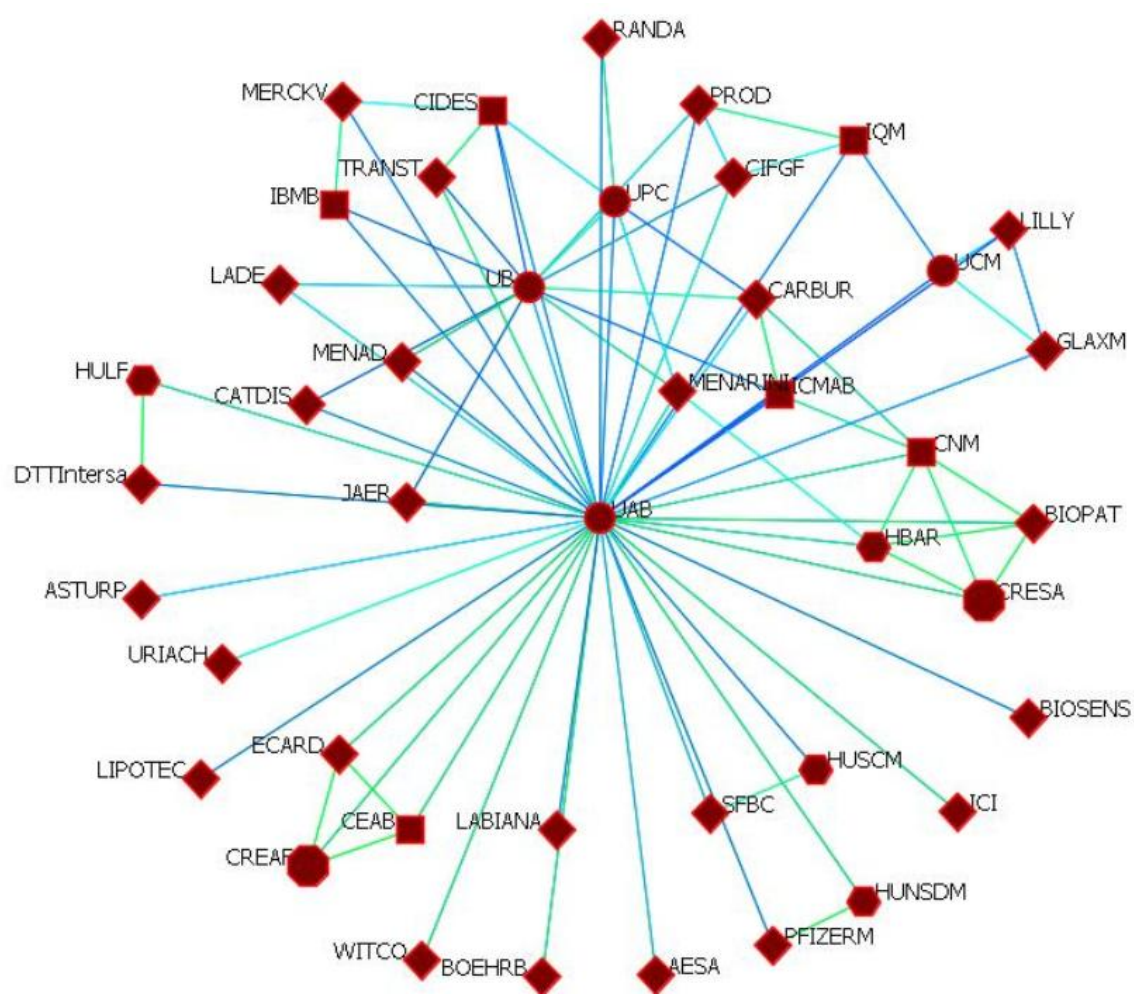

Figura 2. Red egocéntrica de la UAB

La red egocéntrica de la Universidad Complutense de Madrid (UCM) con su vecindario inmediato, nodos que se sitúan a una distancia 1 del ego, se representa en la figura 3. El vecindario inmediato de la red de la UCM está formado por 35 nodos, lo que supone que en términos porcentuales este círculo de influencia es el $7.56 \%$ respecto a la red completa de colaboración entre empresas españolas e instituciones españolas durante el periodo de análisis, vinculados entre sí por un total de 140 relaciones. Esas relaciones tienen más peso cuando ocurren con nodos del sector empresas, que son los nodos más frecuentes en la red egocéntrica de la UCM.

De entre todos los nodos de la red egocéntrica de la UCM, son dependientes del nodo ego un total de cuatro instituciones que no tienen relación en la red con otros nodos diferentes a la UCM y mantienen por tanto relaciones de colaboración exclusivas. Son Bakelite Iberica (BAKEL), Carrefour (CARR), Laboratorios Cayacea (CAYAC) y Laboratorios Farmalider SA (FARMAL), una vez más nodos que en todos los casos pertenecen al sector empresas. 


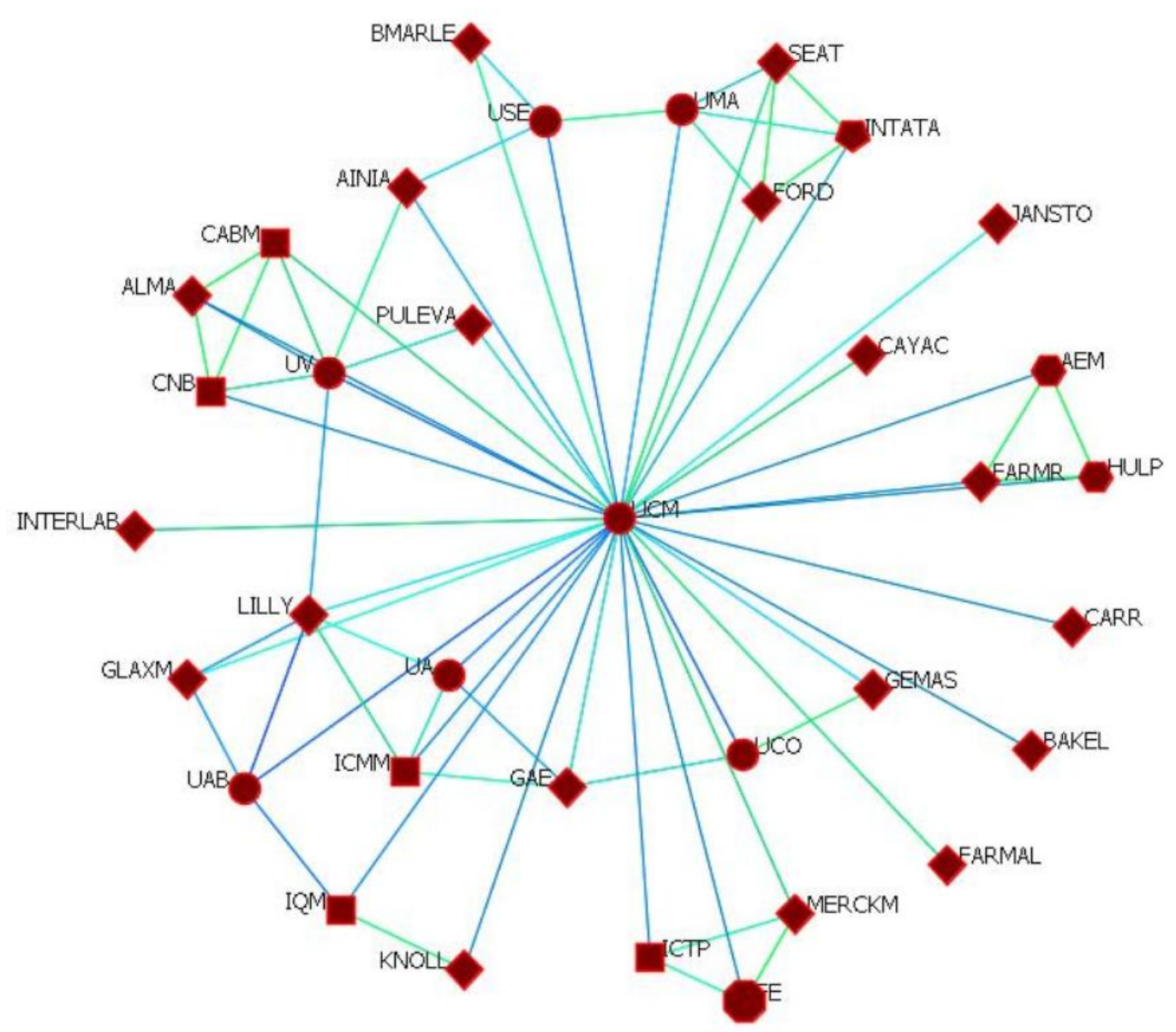

Figura 3. Red egocéntrica de la UCM

\section{Discusión y conclusiones}

Este trabajo comenzaba planteando los tres interrogantes que han guiado el desarrollo de este artículo. Se ha demostrado que es posible establecer la red de influencias entre instituciones involucradas en la producción científica, según los datos de coautoría recogidos en el WoS.

En relación a esas influencias es importante hacer mención a la información incluida en uno de los trabajos que fue pionero en utilizar una frase, y que dará paso a un concepto debatido, que se utiliza dentro del contexto de las organizaciones: la mención al capital social. Fue en el año 1916 cuando el autor Lyda Judson Hanifan ${ }^{3}$, afirmó "si los individuos entran en contacto con sus vecinos, y ellos con otros vecinos, habrá una acumulación de capital social, que puede satisfacer inmediatamente sus necesidades sociales y puede producir un potencial suficiente para realizar una mejora..."

\footnotetext{
${ }^{3}$ Hanifan LJ. (1916) "The Rural School Community Center". En: Annals of the American Academy of Political and Social Science, 1916, (67), pp. 130-138. Citado por Wallis J, Killerby P, Dollery B (2004) "Social Economics and Social Capital". En: International J ournal of Social Economics, 31, 3, pp. 239-258.
} 
Si bien el texto hace referencia al papel a los individuos en un entorno local, cuando se aplica a instituciones, lo que ocurre es que éstas entran en contacto con sus vecinos (nodos a distancia uno) y éstas a su vez con otras. En ese caso, los nodos situados a una distancia geodésica dos del ego, serán los que ofrezcan una oportunidad añadida de acumular mayor capital para satisfacer sus necesidades en aras de una mejora y en beneficio de la propia institución ego.

Así la red que representa las relaciones que ocurren entre las instituciones, es el espacio más adecuado donde se crea y recrea el capital social. Para James Coleman (Coleman JS, 1988) el capital social constituye un recurso cuya particularidad radica en ser algo inherente a la estructura de las relaciones sociales. Este recurso facilita el logro de objetivos o intereses individuales que no podrían alcanzarse en ausencia del capital social al que puede tener acceso por su red o conllevarían un coste muy alto. El mismo autor aclara que el capital social radica en una diversidad de entidades intangibles con dos elementos en común, uno es que todos consisten en algún aspecto de la estructura social y otro es que facilita ciertas acciones de los actores dentro de la estructura.

Las redes de influencias estudiadas comparten la característica sectorial de que sus egos pertenecen a instituciones del sector universitario, consecuencia de la inclusión de éstas en los tres primeros lugares después de realizar el Análisis Factorial que las posiciona como nodos más centrales. Estos tres nodos mantienen las relaciones más intensas y frecuentes con los nodos vinculados al sector mayoritario de esta red, las empresas.

En cuanto a los nodos dependientes, en las redes de influencia de estos egos, son mayoritariamente instituciones del sector empresas que van a vincular al ego mediante un vínculo puente (Granovetter MS, 1973), es decir, una conexión por única y exclusiva, que si se desapareciese haría que el número de componentes de la red se viese incrementado. En ese sentido los nodos dependientes van a tener la posibilidad de acceder a los entornos de instituciones para ellos lejanos o de difícil acceso por sí solos, a través del ego. Si bien el ego con estas relaciones recíprocas pero exclusivas, no podrá hacer que su capital social aumente en la misma proporción que ocurre para los nodos dependientes. 


\section{Bibliografía}

Bonacich P. (1972) "Factoring and weighting approaches to status scores and clique identification". J ournal of Mathematical Sociology, 2 (1), pp. 113-120.

Bordons M; Gómez I. (2000). "Collaboration Networks in Science". En: The Web of Knowledge. A festschrift in honor to Eugene Garfield. New Jersey: Asis, pp. 197213

Börner K; Chen C; Boyack KW. (2003) "Visualizing knowledge domains". Annual Review of Information Science and Technology, 37, pp. 179-225.

Börner K; Sanyal S; Vespignani A. (2007) "Network Science". Annual Review of Information Science and Technology, 41, pp. 537-607.

Brandes U. (2008) "On variants of shortest-path between centrality and their generic computation". Social Networks, 30, pp. 136-145.

Brin S; Page L. 1998. The anatomy of a large-scale hypertextual Web search engine. [En línea]. Disponible en: <http://infolab.stanford.edu/pub/papers/google.pdf> [Fecha de consulta: 21-102010].

Carrasco JA; Miller EJ. (2006) "Exploring the propensity to perform social activities: a social network approach". Transportation, 33 (5), pp. 463-480.

Cea D'Ancona MA. (2002) Análisis multivariable. Teoría y práctica en la investigación social. Madrid: Editorial Síntesis.

Chinchilla Rodríguez Z. Análisis del dominio científico español: 1995 - 2002 (ISI, Web of Science). [Tesis Doctoral]. Granada: Universidad de Granada, 2004.

Coleman JS. (1988) "Social capital in the creation of human capital". American Journal Sociology, 94 (Supplement), pp. S95-S120.

Costa J. (1998) La esquemática: visualizar la información. Barcelona: Paidós.

Cronin B. (2001) "Hyperauthorship: A postmodern perversion or evidence of a structural shift in scholarly communication practices?". Journal of American Society for Information Science and Tecnology, 52 (7), pp. 558-569.

Eades P. (1984) "A heuristic for graph drawing". Congressus Numeratium, 42, pp. 149-160. 
European Comission. (2003) Third European Report on Science \& Technology Indicators.

Fenn DJ; Porter MA; McDonald M; Williams S; Johnson NF; Jones NS. 2009. Dynamic communitites in multichannel data: An application to the foreign exchange market during the 2007-2008 credit crisis. [En línea]. Disponible en: <http://arXiv:0811.3988v3 > [Fecha de consulta: 21-10-2010].

Friedkin NE. (1998) A structural theory of social influence. Cambridge: Cambridge University Press.

Gómez I; Fernández MT; Bordons M; Morillo F. Proyecto de obtención de indicadores de producción científica y tecnológica de España (1996-2001). Consejo Superior de Investigaciones Científicas, 2004.

Granovetter MS. (1973) "The strength of weak ties". American Journal Sociology, 78 (6), pp. 1360-1380.

Hanneman RA. (2005) Introduction to Social Network Methods. Riverside: University of California.

Hara N; Solomon P; Kim SL; Sonnenwald DH. (2003) "An emerging view of scientific collaboration: scientists' perspectives on collaboration and factors that impact collaboration". Journal of American Society for Information Science and Tecnology, 54 (10), pp. 952-965.

Katz JS; Martin BR. (1997) "What is research collaboration?". Research Policy, 26, pp. 1-18.

Kleinberg J M. 1998. Authoritative Sources in a Hyperlinked Environment. [En línea]. Disponible en: <http://www.cs.cornell.edu/home/kleinber/auth.pdf> [Fecha de consulta: 12-10-2010].

Laudel G. (2002) "What do we measure by co-authorships?". Research Evaluation, 11 (1), pp. 3-15.

Luukkonen T; Persson O; Sivertsen G. (1992) "Understanding patterns of international scientific collaboration". Science, Technology, \& Human Values, 17 (1), pp. 101-126.

Mattessich PW; Monsey BR. (1992) Collaboration: What makes it work. A review of research literature on factors influencing successful collaboration. Lafond, St. Paul: Amherst $\mathrm{H}$. Wilder Foundation. 
Melin G; Persson O. (1996) "Studying research collaboraion using co-authorships". Scientometrics, 36 (3), pp. 363-377.

Milojevic S. (2010) "Modes of collaboration in modern science: beyond power laws and preferential attachment". Journal of American Society for Information Science and Tecnology, 61 (7), pp. 1410-1423.

Moed HF. (2005) Citation Analysis in Research Evaluation. Holanda: Springer.

Molina JL. (2005) "El estudio de las redes personales: contribuciones, métodos y perspectivas". EMPIRIA.Revita de Metodología en Ciencias Sociales, 10, pp. 71-105.

Molina J L; Muñoz J M; Domenech M. Redes de publicaciones científicas: una análisis de la estructura de coautorías. Sunbelt XXI Budapest 25-28 abril 2001: 2001,

Moya Anegón F; Chinchilla Rodríguez Z. (2004) Indicadores bibliométricos de la actividad científica española. (ISI, Web of Science, 1998-2002). Madrid: FECYT.

Moya Anegón F; Chinchilla Rodríguez Z. (2005) Indicadores bibliométricos de la actividad científica española - 2004. Madrid: FECYT.

Moya Anegón F; Vargas Quesada B; Chinchilla Rodríguez Z; Corera Álvarez E; Herrero Solana V; Muñoz Fernández F. (2005) "Domain analysis and information retrieval through the construction of heliocentric maps based on ISI-JCR category cocitation". Information Processing \& Management, 41, pp. 1520-1533.

Moya Anegón F (dir.); Chinchilla Rodríguez Z; Corera Álvarez E; Gómez-Crisóstomo R; González Molina A; Muñoz Fernández F, [et al.] . (2007) Indicadores bibliométricos de la Actividad Científica Española (1990-2004). Madrid: FECYT.

Nooy W; Mrvar A; Batagelj V. (2004) Exploratory Social Network Analysis with Pajek. New York: Cambridge University Press. (Estructural Analysis in the Social Sciences, 27)

Olmeda Gómez C; Perianes Rodríguez A; Ovalle Perandones MA; Moya Anegón F. (2008) "Comparative analysis of university-government-enterprise co-authorship networks in three scientific domains in the region of Madrid". Information Research, 13 (3), pp. Paper 352

Perianes Rodríguez A; Olmeda Gómez C; Moya Anegón F. (2010) Redes de colaboración científica. Valencia: Tirant Online.

Russell JM; Madera-Jaramillo MJ; Ainsworth S. (2009) "El análisis de redes en el estudio de la colaboración científica". REDES- Revista Hispana para el análisis de redes sociales, $17(2)$, 
Smith D; Katz JS. Collaboratie Approaches to Research. 2000.

Subramanyam K. (1983) "Bibliometric studies of research collaboration: a review". J ournal of Information Science, 6, pp. 33-38.

Vargas Quesada B; Moya Anegón F; Chinchilla Rodríguez Z; Corera Álvarez E; Guerrero Bote V. (2008) "Evolución de la estructura científica española: ISI Web of Science 1990-2005". El Profesional de la Información, 17 (1), pp. 22-37.

Wasserman S; Faust K. (1994) Social Network Analysis. Structural Analysis in the Social Sciences 8

Wellman B. (1988). "Structural analysis: from method and metaphor to theory and substance". En: Wellman B and Berkowit SD. Social structures: a network approach. Cambridge: Cambridge Unviersity Press, pp. 19-61

White HC. (2001) "Author-centered bibliometrics through CAMEOs: Characterizations automatically made and edited online". Scientometrics, 51 (3), pp. 607-637.

White HC; McCain KW. (1997) "Visualization of literatures". Annual Review of Information Science and Technology, 32, pp. 99-168.

Zitt M; Bassecoulard E; Okubo Y. (2000) "Shadows of the past in international cooperation: collaboration profiles of the top five producers of science". Scientometrics, 47 (3), pp. 627-657. 


\section{Anexo}

\begin{tabular}{|c|c|}
\hline Abreviatura & Institución \\
\hline ABTOM & Abbott Científica SA \\
\hline ACERI & Acerinox SA \\
\hline ADVAN & Advancell \\
\hline AEM & Agencia Española del Medicamento \\
\hline AESA & Asfaltos Españoles SA \\
\hline AINIA & Instituto Tecnológico Agroalimentario AI NIA \\
\hline ALMA & Alma Bioinformática SL \\
\hline ASTURP & Asturpharma SA \\
\hline ATMSA & Asistencia Tecnológica Medioambiental SA \\
\hline BAKEL & Bakelite Iberica \\
\hline BAYERB & Farmaceútica Bayer SA \\
\hline BIOPAT & Biopat \\
\hline BIOSENS & Biosensores SL \\
\hline BMARLE & Instituo Biomar SA \\
\hline BOEHRB & Boehringer Ingelheim España SA \\
\hline CABM & Centro de Astrobiología \\
\hline CARBUR & Carburos Metálicos SA \\
\hline CARR & Carrefour \\
\hline CATDIS & Catalana de Dispensación SA \\
\hline CAYAC & Laboratorios Cayacea \\
\hline CEAB & Centro d'Estudis Avancats de Blanes \\
\hline CENAVI & Laboratorios Cenavisa SA \\
\hline CERTALL & Certa \\
\hline CHANDON & Masia Chandon \\
\hline CIDES & Centro Investigación \& Desarrollo \\
\hline ClFGF & Centro Investigación Grupo Ferrer \\
\hline CNB & Centro Nacional de Biotecnología \\
\hline CNM & Centro Nacional Microelectrónica \\
\hline CREAF & Centre de Recerca Ecològica i Aplicacions Forestals \\
\hline CRESA & Fundación Centre de Recerca en Sanitat Animal \\
\hline CYGEN & Cygene Spain SL \\
\hline DTTintersa & Dietéticos Intersa SA \\
\hline ECARD & Cardedeu Field Station \\
\hline EKO & Ekulor Asesoría Ambiental SL \\
\hline ENANT & Enantia SL \\
\hline EUROM & Euromed SA \\
\hline FARMAL & Laboratorios Farmalider SA \\
\hline FARMR & Laboratorios Farmalider Madrid SA \\
\hline FE & Filmoteca Española \\
\hline
\end{tabular}




\begin{tabular}{|c|c|}
\hline Abreviatura & Institución \\
\hline FORD & Ford \\
\hline FREIXE & Freixenet SA \\
\hline GAE & Grupo Antolin Ingeniería SA \\
\hline GAIKER & Centro Tecnológico Gaiker \\
\hline GastroVasc & Gastronomía Vasca \\
\hline GEMAS & Gemasur \\
\hline GLAXM & Glaxo Wellcome \\
\hline HBAR & Hospital de Barcelona \\
\hline HCPB & Hospital Clínico y Provincial de Barcelona \\
\hline HGUVH & Hospital General Universitario Vall d'Hebron \\
\hline HUDJT & Hospital Universitario de Girona Dr Josep Trueta \\
\hline HULF & Hospital Universitario La Fe de Valencia \\
\hline HULP & Hospital Universitario de La Princesa \\
\hline HUNDSDM & Hospital de Nuestra Señora del Mar \\
\hline HUSCM & Hospital Clínico Universitario San Carlos \\
\hline IBMB & Institut de Biología Molecular de Barcelona \\
\hline ICATTEC & Instituto Catalán de Tecnología \\
\hline $\mathrm{ICl}$ & $\mathrm{ICI}$ Spain SA \\
\hline ICMAB & Institut Ciència Materials de Barcelona \\
\hline ICMM & Instituto de Ciencia Materiales de Madrid \\
\hline ICTP & Instituto de Ciencia y Tecnología de los Polímeros \\
\hline IDIBAPS & Instituto Investigación Biomédica August Pi i Sunyer \\
\hline IEMA & Instituto de Estructura de la Materia \\
\hline IIQAB & Instituto Investigación Química \& Ambientals Barcelona J Pascal \\
\hline INTATA & Inta \\
\hline INTERLAB & Grupo Interlab SA \\
\hline IQM & Instituto de Óptica Daza de Valdés \\
\hline IRBB & Institut de Recerca Biomédica de Barcelona \\
\hline IUSC & Centro de formación IUC \\
\hline JAER & Laboratorio Jaer SA \\
\hline JANSTO & Hanssen Cilag \\
\hline KNOLL & Laboratorio Knoll SA \\
\hline LABIANA & Labiana Life Scientific SA \\
\hline LADE & Laboratorio del Dr Esteve SA \\
\hline LASA & Laboratorio Lasa SA \\
\hline LESVI & Laboratorio Lesvi SL \\
\hline LILLY & Laboratorio Lilly SA \\
\hline LIPOTEC & Lipotec SA \\
\hline LUCTA & Lucta SA \\
\hline MCDERM & MacDermid Española SA \\
\hline MENAD & Menadiona SA \\
\hline MENARINI & Menarini Diagnost \\
\hline MERCKM & Labs Merck Sharp \& Dohme España SA \\
\hline
\end{tabular}




\begin{tabular}{|c|c|}
\hline Abreviatura & Institución \\
\hline MERCKV & Merck Farma y Química SA \\
\hline MONOC & Monocrom SL \\
\hline NUTREXPA & Nutrexpa SA \\
\hline ORDESA & Laboratorios Ordesa \\
\hline PARCI & Parc Científic Barcelona \\
\hline PFIZERM & Pfizer SA \\
\hline PHARMAR & PharmaMar SA \\
\hline PREMA & Prema Compañía SA \\
\hline PROD & Almirall Prodesfarma SA \\
\hline PULEVA & Puleva SA \\
\hline PYMAG & Pymag SA \\
\hline RANDA & Randa Group SA \\
\hline REPSOLM & Respol \\
\hline RMN & Laboratorio RMN Biomol \\
\hline SALVAT & Laboratorios Salvat SA \\
\hline SEAT & Seat SA \\
\hline SEGURA & Segura Viudas SAU \\
\hline SFBC & SFBC Anapharm Europe \\
\hline TRANST & Transtechnology España SL \\
\hline UA & Universidad de Alicante \\
\hline UAB & Universidad Autónoma de Barcelona \\
\hline UB & Universitat de Barcelona \\
\hline UCM & Universidad Complutense de Madrid \\
\hline UCO & Universidad de Córdoba \\
\hline UH10 & Hospital Universitario 12 de Octubre \\
\hline UM & Universidad de Murcia \\
\hline UMA & Universidad de Málaga \\
\hline UPC & Universitat Politécnica de Cataluña \\
\hline URIACH & Centro de Investigación J Uriach y Cía SA \\
\hline URL & Universitat Ramón Llul \\
\hline URV & Universitat Rovira i Virgili \\
\hline USE & Universidad de Sevilla \\
\hline UV & Universidad de Valencia \\
\hline VALCEM & Valenciana de Cementos Portland SA \\
\hline VITA & Laboratorio Vita-I nvest SA \\
\hline WalPack & Walter Pack \\
\hline WITCO & Witco España SL \\
\hline
\end{tabular}

Tabla 2. Abreviaturas e instituciones 